\title{
State Observer-Based Backstepping Sliding Mode Control for Electro-Hydraulic Systems
}

\author{
Phan Viet Tan \\ Hanoi, Vietnam \\ Cuong Nguyen Manh
Hanoi, Vietnam
}

\author{
Nguyen Quang Duc \\ Hanoi, Vietnam
}

\author{
Danh Huy Nguyen \\ Hanoi, Vietnam \\ huy.nguyendanh@hust.edu.vn
}

\author{
Le Ngoc Minh \\ Hanoi, Vietnam \\ minh.ln181646@sis.hust.edu.vn \\ Tung Lam Nguyen \\ Hanoi, Vietnam \\ lam.nguyentung@hust.edu.vn
}

\begin{abstract}
In the paper, a control method using backstepping integrated with sliding mode control based on high-gain observer is proposed for an Electro-Hydraulic system (EHS). The observer is dedicated to estimate full-state variables by using output position feedback. In the initial step, the control design formula is constructed via backstepping technique. Following that, the control signal is designed based on sliding mode control (SMC) algorithm, and thus lead to the controller that has the advantages of these two control laws.
\end{abstract}

Index Terms - component, backstepping, high-gain observer, electro-hydraulic system (EHS), sliding mode.

\section{INTRODUCTION}

$\mathrm{N}$ OWADAYS, various useful applications related to electro-hydraulic system (EHS), including manipulators [1], anti-lock braking systems [2], hydraulic excavator [3] and plate hot rolling [4] are extremely important in modern industrial automation due to its distinct advantages: higher load stiffness, superior load efficiency and high power-to-weight ratio.

However, EHSs exhibit significant dynamical nonlinearities such the complicated flow properties of servo valves. Numerous control methods have been studied and proposed to achieve the acceptable control quality - such as the local linearization of nonlinear dynamics in [5]. Practically, to overcome the control design's challenges in precisely measuring the state parameters or the disturbance through sensors due to the limitation of space and cost constraint, there is a trend in using a variety of different observers, mentioned in [6] and [7]. Recently, the high-gain state observer is proposed to estimate the state parameters, and especially, one can tune the observer bandwidth to obtain the desired stability/robustness properties [6]. For the control technique, many have used PID [8], backstepping [7], and sliding mode [9]. One of the most common approaches is backstepping method, it was applied to EHS [6] for achieving exponential convergence of the system position and load pressure. But in some cases, the control law using this technique is sensitive to noise and the higher the order of the system, the more complex the calculation steps, hence reduces the response speed of the system.

Sliding mode control approach is also a suitable alternative due to the significant efficiency in stabilizing the posi- tion controlling in the changes of the system [9]. It gives us a much more quality performance when there was appearance of disturbance but meets difficulties with massive calculation and "the chattering effect" leading to considerable wear of moving mechanical parts and high heat lost in power circuits [10].

In this study, a high-gain state observer integrated backstepping control with an improvement in combining the sliding mode control is designed to enhance the quality in position tracking. By using the state parameters estimated from the observer and applying a hybrid of backstepping and sliding mode control, the proposed control achieves good system performances.

\section{Electro-Hydraulic System model formulation}

The physical equations describing the EHSs is (Figure 1) presented in detail in [7] and briefly given as

$$
\left\{\begin{array}{l}
\frac{d \underline{x}}{d t}=A \underline{x}+\underline{\gamma}(\underline{x}, u, y) \\
y=C \underline{x}
\end{array}\right.
$$

$$
\begin{aligned}
& \text { Where: } A=\left[\begin{array}{lll}
0 & 1 & 0 \\
0 & 0 & 1 \\
0 & 0 & 0
\end{array}\right] \text { and } \\
& \begin{aligned}
\underline{\gamma}(\underline{x}, u, y) & =\left[\begin{array}{c}
0 \\
k_{1} x_{1}+k_{2} x_{2}+\left(k_{3}-1\right) x_{3}+d_{2} \\
g_{1} x_{2}+g_{2} x_{3}+d_{3}+g_{3} u
\end{array}\right] \\
& =\left[\begin{array}{lll}
0 & \gamma_{2} & \gamma_{3}
\end{array}\right]^{T}
\end{aligned}
\end{aligned}
$$

$\underline{\gamma}(\underline{x}, u, y)$ satisfies Locally Lipschitz Condition in the following compact set:

$$
\|\underline{\gamma}(\underline{x}, u, y)-\underline{\gamma}(\underline{\hat{x}}, u, y)\| \leq a\|\underline{x}-\underline{\hat{x}}\| \quad \forall \underline{x}, \underline{\hat{x}}, u, y
$$

where $a$ is called Lipschitz constant and $C=\left[\begin{array}{lll}1 & 0 & 0\end{array}\right]$ and: 


$$
\begin{gathered}
k_{1}=-\frac{k}{m} ; k_{2}=-\frac{b}{m} ; k_{3}=\frac{A_{p}}{m} ; g_{1}=-h_{1} ; g_{2}= \\
-h_{2} ; g_{3}=h_{3} \sqrt{P_{s}-\operatorname{sgn}(u) x_{3}} \\
d_{2}=-\frac{F_{L}}{m}-\frac{F_{F}}{m}+\Delta f_{2} ; d_{3}=\Delta f_{3}
\end{gathered}
$$

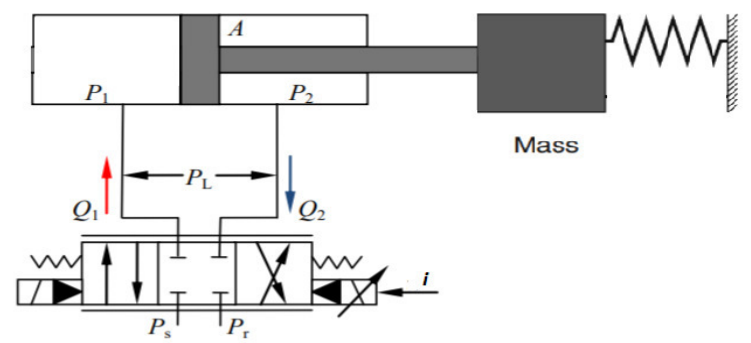

Fig. 1. A Representative EHS

\section{HGGSOB - BASED BACKSTEPPING SLIDING ModE CONTROL}

\section{A. High-gain States Observer Design}

To approximate $\underline{x}$ we propose a state observer as follow:

$$
\frac{d \underline{\underline{x}}}{d t}=A \underline{\hat{x}}+\gamma(\underline{\hat{x}}, u, y)+L(y-\hat{y})
$$

where $\hat{x}$ is the state estimation and $L=\left[\begin{array}{lll}l_{1} & l_{2} & l_{3}\end{array}\right]^{T}$ is the observer gain, chosen such that $(A-L C)$ has all real parts of eigenvalues in the left half plane, constant matrix $L$ is given as follows $L=\left[\begin{array}{lll}h_{1} \varepsilon^{-1} & h_{2} \varepsilon^{-2} & h_{3} \varepsilon^{-3}\end{array}\right]^{T}$, where $\varepsilon$ is small positive number and $h_{1}, h_{2}, h_{3}$ are coefficient of the Hurwitz polynomial.

\section{B. Backstepping-Sliding Mode Control design}

We define the position of piston/output, velocity of piston and pressure difference tracking error as $e_{1}, e_{2}, e_{3}$. Let us start by defying the first Lyapunov candidate function as:

$$
V_{1}=\frac{1}{2} e_{1}^{2}
$$

The derivative of the $V_{1}$ is given by:

$$
\dot{V}=e_{1} \dot{e}_{1}=e_{1}\left(x_{2}-\dot{x}_{1 d}\right)
$$

We choose $x_{2 d}=\dot{x}_{1 d}-p_{1} e_{1}$ where $p_{1}$ is a positive number, then:

$$
\dot{V}=-p_{1} e_{1}^{2}+e_{1} e_{2}
$$

Let us define $V_{2}$ as:

$$
V_{2}=V_{1}+\frac{1}{2} e_{2}{ }^{2}
$$

Then

$$
\begin{aligned}
\dot{V}_{2} & =-p_{1} e_{1}{ }^{2}+e_{1} e_{2}+e_{2} \dot{e}_{2} \\
& =-p_{1} e_{1}{ }^{2}+e_{2}\left(e_{1}+\dot{e}_{2}\right)
\end{aligned}
$$

From (7), $\dot{V}_{2}$ can be rewritten as:

$$
\dot{V}_{2}=-p_{1} e_{1}{ }^{2}+e_{2}\left(e_{1}+k_{1} x_{1}+k_{2} x_{2}+k_{3} x_{3}+d_{2}-\dot{x}_{2 d}\right)
$$

Let choose:

$$
x_{3 d}=\frac{1}{k_{3}}\left(-e_{1}-k_{1} x_{1}-k_{2} x_{2}-d_{2}+\dot{x}_{2 d}-p_{2} e_{2}\right)
$$

where $p_{2}$ is a strict positive gain, this results in:

$$
\dot{V}_{2}=-p_{1} e_{1}{ }^{2}-p_{2} e_{2}{ }^{2}+k_{3} e_{2} e_{3}
$$

Assuming $e_{3}$ will be driven to zero, it can be shown that:

$$
\dot{V}_{2}=-p_{1} e_{1}{ }^{2}-p_{2} e_{2}{ }^{2} \leq 0
$$

Now $u$ is chosen to drive $e_{3}$ to zero, we define sliding surface $S_{3}$ as follow:

$$
S_{3}=x_{3}-x_{3 d}
$$

Differentiating sliding surface $S_{3}$ yields:

$$
\begin{aligned}
\dot{S}_{3} & =\dot{x}_{3}-\dot{x}_{3 d} \\
& =g_{1} x_{2}+g_{2} x_{3}+g_{3} u+d_{3}-\dot{x}_{3 d}
\end{aligned}
$$

The control signal $u$ can be divided to two components: $u_{e q}$ will drive sliding surface to zero and $u_{s w}$ will lead the system states on the sliding surface thus the control signal can be written as:

$$
u=u_{e q}+u_{s w}
$$

From (28), it is straightforward to show that:

$$
u_{e q}=\frac{1}{g_{3}}\left(-g_{1} x_{2}-g_{2} x_{3}-d_{3}+\dot{x}_{3 d}\right)
$$

In order to have $S_{3} \rightarrow 0$, we need to design signal $u_{s w}$ renders $S_{3} \dot{S}_{3}<0$, so $u_{s w}$ can be selected as follow:

$$
u_{s w}=\frac{1}{g_{3}}\left(-m_{1} \operatorname{sign}\left(S_{3}\right)-m_{2} S_{3}\right)
$$

where $m_{1}, m_{2}$ are positive constants. With the designed control signal, the stability of the system will be considered according to the Lyapunov stable standards. The third Lyapunov candidate function is proposed to prove the stability of the whole system:

$$
V_{3}=\frac{1}{2} S_{3}^{2}
$$

Taking time derivative of $V_{3}$ results in:

$$
\dot{V}_{3}=-m_{1} S_{3} \operatorname{sign}\left(S_{3}\right)-m_{2} S_{3}{ }^{2} \leq 0
$$

Finally, the control law can be written as:

$$
\left\{\begin{array}{l}
u=\frac{1}{g_{3}}\left(g_{1} x_{2}+g_{2} x_{3}-d_{3}+\dot{x}_{3 d}\right)+\frac{1}{g_{3}}\left(-m_{1} \operatorname{sign}\left(S_{3}\right)-m_{2} S_{3}\right) \\
x_{2 d}=\dot{x}_{1 d}-p_{1} e_{1} \\
x_{3 d}=\frac{1}{k_{3}}\left(-e_{1}-k_{1} x_{1}-k_{2} x_{2}-d_{2}+\dot{x}_{2 d}-p_{2} e_{2}\right)
\end{array}\right.
$$

\section{Simulation Results}

In the simulation and experiments (using MATLAB version 2018a), the following parameters were used: $m_{1}=$ $300, m_{2}=300, p_{1}=150, p_{2}=600, \varepsilon=1 \mathrm{e}-6$ $L=\left[\begin{array}{lll}3 \varepsilon^{-1} & 3 \varepsilon^{-2} & 1 \varepsilon^{-3}\end{array}\right]^{T}$. In the simulation, to examine the performance of the closed-loop system with the proposed method, the following case is considered with disturbances are parameter uncertainties, $d_{2}=-0.3 \times f_{2}$ and $d_{3}=-0.3 \times f_{3}$

\section{A. Constant reference tracking performance}




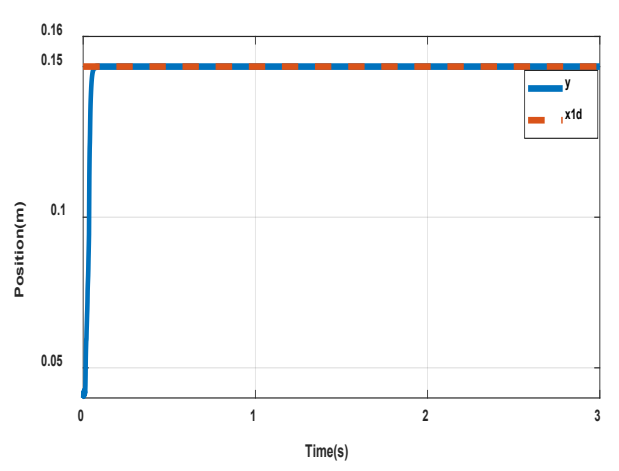

Fig. 4.1. Backstepping integrated with sliding mode control

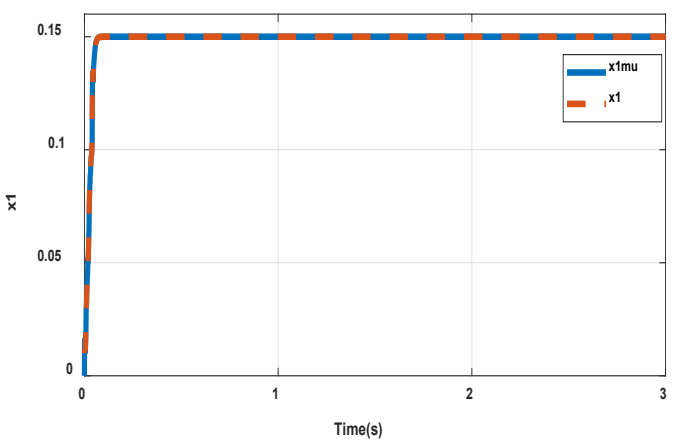

Fig. 4.2. Estimation performance of $\mathrm{x}_{1}$

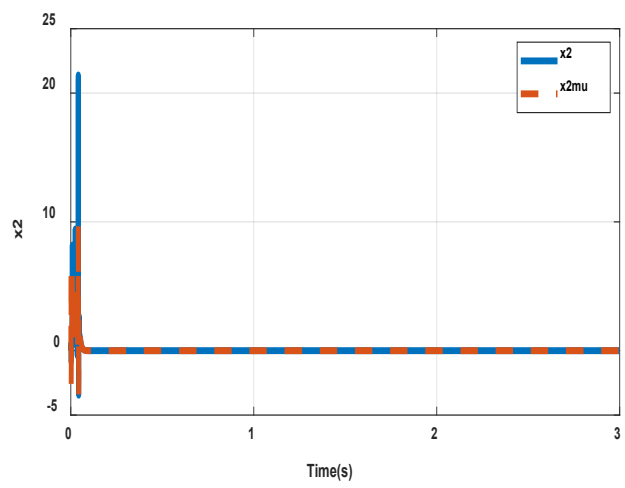

Fig. 4.3. Estimation performance of $\mathrm{x}_{2}$

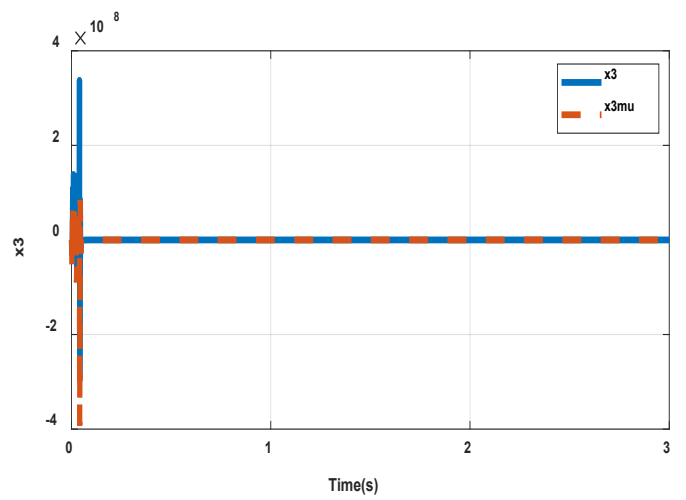

Fig. 4.4. Estimation error of $x_{3}$

Fig. 4.1 exhibit the position tracking errors. As we see, the position of the piston approaches quickly and well-tracking to the desired output signal. The proposed method exhibits zero position tracking error. Fig. 4.2, 4.3, and 4.4 show that the state variables were well estimated in a moment.

B. Comparison to conventional backstepping control

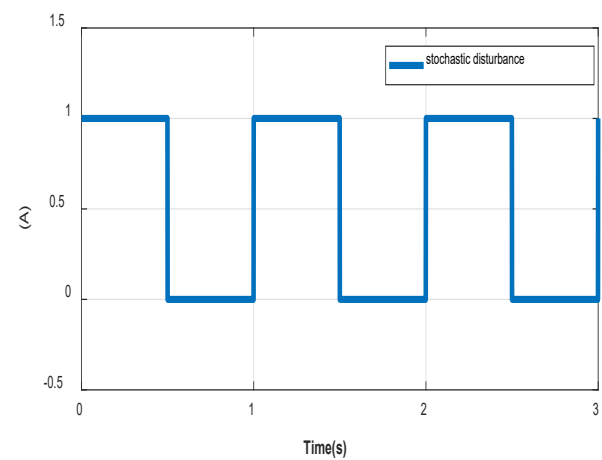

Fig. 4.5. Stochastic disturbance with pulse appearance

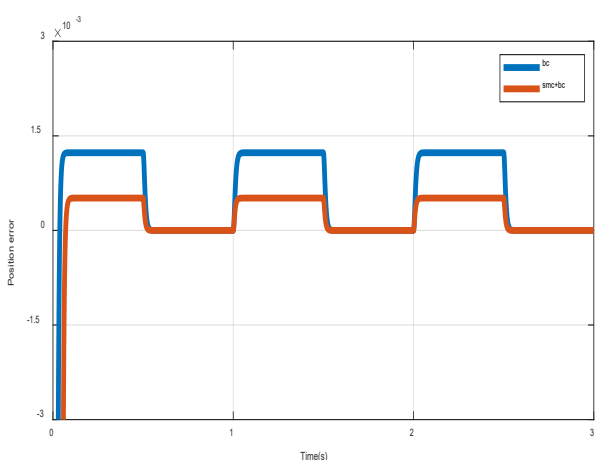

Fig. 4.6. Tracking error of two method with high gain state observer.

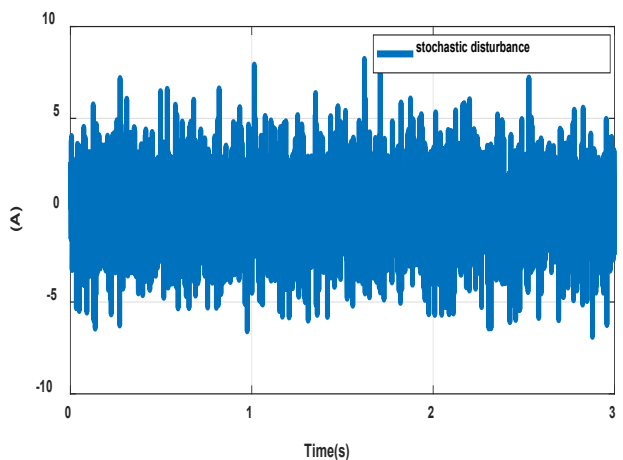

Fig 4.7. Stochastic disturbance in white noise appearance

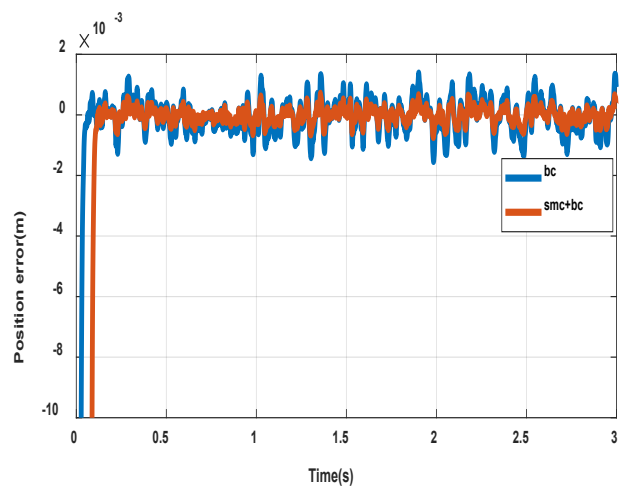

Fig. 4.8. Tracking error of two method with high gain state observer and constant input 
In two cases, the proposed method always has a smaller position error characteristic than conventional backstepping controller. It shows that the proposed method helps stabilize effectively when the stochastic disturbances present in some part of EHSs.

\section{CONCLUSION}

The HGSOB-based backstepping aggregated sliding mode control for EHSs was proposed to guarantee the position tracking performance in the face of stochastic disturbances. The HGSOB was designed to estimate full-state variables with very high accuracy. The sliding mode control help EHSs stable with stochastic disturbances in several part of the system, while backstepping force the output signal rapidly approaching to desired signal. The simulation indicates that the proposed methods improve the position tracking performance. In the future, we are looking forward to conducting more research on parallel robots.

\section{ACKNOWLEDGMENT}

This research was funded by Hanoi University of Science and Technology grant number T2020-PC-022.

\section{REFERENCES}

[1] Zhu, W.-H., \& Piedboeuf, J.-C. (2005). Adaptive Output Force Tracking Control of Hydraulic Cylinders with Applications to Robot Manipulators. Journal of Dynamic Systems, Measurement, and Control.
[2] Liu, T., Yu, Z., Xiong, L., \& HAN, W. (2017). Anti-Lock Braking System Control Design on An Integrated-Electro-Hydraulic Braking System. SAE International Journal of Vehicle Dynamics, Stability, and $\mathrm{NVH}$.

[3] Le Hanh, D., Ahn, K. K., Kha, N. B., \& Jo, W. K. (2009). Trajectory control of electro-hydraulic excavator using fuzzy self-tuning algorithm with neural network. Journal of Mechanical Science and Technology, 23(1), 149-160.

[4] Kim, M. Y., \& Lee, C.-O. (2006). An experimental study on the optimization of controller gains for an electro-hydraulic servo system using evolution strategies. Control Engineering Practice, 14(2), 137147.

[5] Kim, W., Won, D., Shin, D., \& Chung, C. C. (2012). Output feedback nonlinear control for electro-hydraulic systems. Mechatronics, 22(6), 766-777.

[6] Won, D., Kim, W., Shin, D., \& Chung, C. C. (2015). High-Gain Disturbance Observer-Based Backstepping Control with Output Tracking Error Constraint for Electro-Hydraulic Systems. IEEE Transactions on Control Systems Technology, 23(2), 787-795.

[7] Guo, Q., Zhang, Y., Celler, B. G., \& Su, S. W. (2016). Backstepping Control of Electro-Hydraulic System Based on Extended-StateObserver with Plant Dynamics Largely Unknown. IEEE Transactions on Industrial Electronics, 63(11), 6909-6920.

[8] Samakwong, T., \& Assawinchaichote, W. (2016). PID Controller Design for Electro-hydraulic Servo Valve System with Genetic Algorithm. Procedia Computer Science, 86, 91-94.

[9] Guan, C., \& Pan, S. (2008). Adaptive sliding mode control of electrohydraulic system with nonlinear unknown parameters. Control Engineering Practice, 16(11), 1275-1284.

[10] Utkin, V., \& Hoon Lee. (n.d.). Chattering Problem in Sliding Mode Control Systems. International Workshop on Variable Structure Systems, 2006. VSS'06. 